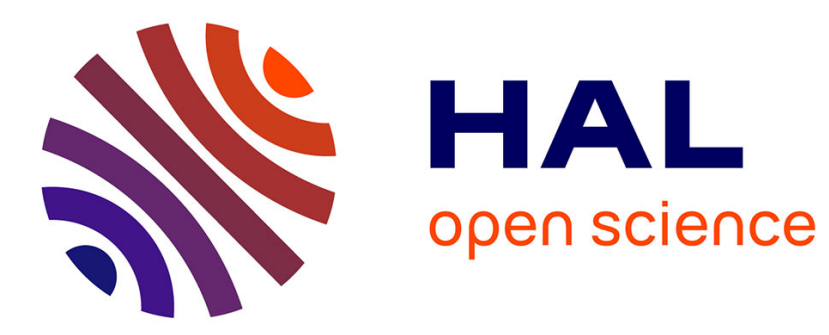

\title{
Statistical Properties of Random Dynamical Systems with Contracting Direction
}

Davide Faranda, Jorge Milhazes Freitas, Pierre Guiraud, Sandro Vaienti

\section{To cite this version:}

Davide Faranda, Jorge Milhazes Freitas, Pierre Guiraud, Sandro Vaienti. Statistical Properties of Random Dynamical Systems with Contracting Direction. Journal of Physics A: Mathematical and Theoretical, 2016, 49 (20), pp.204001. 10.1088/1751-8113/49/20/204001 . hal-01258390

\section{HAL Id: hal-01258390 https://hal.science/hal-01258390}

Submitted on 18 Jan 2016

HAL is a multi-disciplinary open access archive for the deposit and dissemination of scientific research documents, whether they are published or not. The documents may come from teaching and research institutions in France or abroad, or from public or private research centers.
L'archive ouverte pluridisciplinaire HAL, est destinée au dépôt et à la diffusion de documents scientifiques de niveau recherche, publiés ou non, émanant des établissements d'enseignement et de recherche français ou étrangers, des laboratoires publics ou privés. 


\title{
Statistical Properties of Random Dynamical Systems with Contracting Direction
}

\author{
Davide Faranda * $\quad$ Jorge Milhazes Freitas $^{\dagger} \quad$ Pierre Guiraud ${ }^{\ddagger}$ Sandro Vaienti ${ }^{\S}$
}

October 31, 2015

\begin{abstract}
We present a mostly numerical investigation on randomly perturbed piecewise contracting maps (PCM) with the goal to study the extreme value limit distribution of observables related to local recurrence. Our analysis will focus on PCM under additive noise, but we will also consider the hyperbolic attractor of the Baker's map when perturbed with another kind of noise, namely, the randomly applied stochastic perturbation. A comparison of the two kind of noises will be considered with respect to the computation of the extremal index.
\end{abstract}

Keywords: Piecewise contracting maps; Baker map; random dynamical systems; extreme value theory; extremal index; transfer operator.

\section{Introduction}

Previously [6, 7], we showed that piecewise contracting maps (PCM) exhibit classical Extreme Value Laws (EVLs), when they are randomly perturbed. In particular we studied in deep detail what we called Randomly Applied Stochastic Perturbations (RASP). They belong to the broader class of random transformations that we are going to define and they revealed to be particularly useful in the context of PCM for the explicit computation of a few important quantities in the random setting: absolutely continuous stationary measures, transfer operator, rate of mixing, short returns. We were therefore able to show that for large class of PCM perturbed with the RASP and for observables depending upon the choice of at most countably many points, one gets the Gumbel's law almost everywhere but around the attractor of the unperturbed system, where the limiting exponential law is modified by the presence of an extremal index. This was rather surprising because usually random perturbations make all points statistically equivalent. On the other hand RASP is a very special kind of perturbation since it operates an aleatory reset of the state of the dynamical system at each failure of a Bernoulli random variable. It is therefore interesting to ask whether EVLs emerge whenever PCM are perturbed with other kind of noises, in particular with the additive noise. We partially investigated such a question in [6]; we now present here a more wide study, mostly numerical, but supported by formal arguments based on the theory of weakly constrictive (transfer) operators. We recall that with such a theory Lasota and Mackay [14] were already able to get a few statistical properties of PCM perturbed with noise. In here we will focus on the extremal properties of such perturbed systems.

Recently the results of the Extreme Value Theory have brought new techniques that allow to quantify the geometrical and dynamical properties of a certain class of systems. In the case of absolutely continuous invariant measures (acim), precise analytical results can be obtained in terms of classical Extreme Value

${ }^{*}$ LSCE, Laboratoire du Science du Climat et de l'Environment, UMR 8212, CNRS-CEA-UVSQ, 91191 Gif-sur-Yvette, France. e-mail: <davide.faranda@lsce.ipsl.fr>.

†Centro de Matemática \& Faculdade de Ciências da Universidade do Porto, Rua do Campo Alegre 687, 4169-007 Porto, Portugal. e-mail:<jmfreita@fc.up.pt>.

${ }^{\ddagger}$ Centro de Investigación y Modelamiento de Fenómenos Aleatorios - Valparaíso, Facultad de Ingeniería de la Universidad de Valparaíso, General Cruz 222, Valparaíso, Chile. e-mail: <pierre.guiraud@uv.cl>.

$\S$ Aix Marseille Université, CNRS, CPT, UMR 7332, 13288 Marseille, France and Université de Toulon, CNRS, CPT, UMR 7332, 83957 La Garde, France. e-mail:<vaienti@cpt.univ-mrs.fr>. 
Laws and depend on the fulfillment of general mixing conditions and on the observables considered. In fact, those observables are designed in such a way that extreme events are equivalent to detect the recurrence of an orbit to a neighborhood of a given point in the phase space. A collection of such events, under appropriate normalization, is distributed according to one of the three classical EVLs, namely the Gumbel, the Fréchet and the Weibull distributions. The values of the normalizing constants are linked to the local behavior of the measure and, in the case of chaotic dynamics and of the measure being absolutely continuous, they depend only on the number of extremes extracted and on the phase space dimension.

We have already shown in a preceding article [8] that random perturbations of regular systems, in particular rotations, induce the appearance of extreme value laws since the perturbed systems acquire a chaotic behavior. We pursue the same objective here by considering piecewise contracting maps perturbed with additive noise. Although the main focus of this paper is on piecewise contracting maps, we will consider in the last section random perturbations of the hyperbolic attractor of the Baker's map. This map is in fact another interesting test to compare the additive noise with the RASP perturbation. In the paper [7], we presented a general theory of RASP perturbation for a large class of attractors including the limit sets of PCM and the invariant sets of discontinuous hyperbolic maps like the Baker one. Even in the latter case the computation of the extremal index is sensitive to the kind of perturbation we used, in particular the extremal index converges to 1 around periodic points for additive noise, but it persists to be less than 1 for the RASP perturbation.

\section{Random transformations}

Let us consider a sequence of i.i.d. random variables $\left(W_{k}\right)_{k \in \mathbb{N}}$ with values $\left(\omega_{k}\right)_{k \in \mathbb{N}}$ in a space $\Omega_{\varepsilon}$ and with common probability distribution $\theta_{\varepsilon}$. Let $X \subset \mathbb{R}^{d}$ be a compact set equipped with the Lebesgue measure $m$ defined on the Borel $\sigma$-algebra, and $\left(f_{\omega}\right)_{\omega \in \Omega_{\varepsilon}}$ a family of measurable transformations such that $f_{\omega}: X \rightarrow X$ for all $\omega \in \Omega_{\varepsilon}{ }^{1}$. Given a point $x \in X$ and a realization $\underline{\omega}=\left(\omega_{1}, \omega_{2}, \ldots\right) \in \Omega_{\varepsilon}^{\mathbb{N}}$ of the stochastic process $\left(W_{k}\right)_{k \in \mathbb{N}}$, we define the random orbit of $x$ as the sequence $\left(f_{\underline{\omega}}^{n}(x)\right)_{n \in \mathbb{N}}$, where

$$
f_{\underline{\omega}}^{0}(x)=x \quad \text { and } \quad f_{\underline{\omega}}^{n}(x)=f_{\omega_{n}} \circ f_{\omega_{n-1}} \circ \cdots \circ f_{\omega_{1}}(x) \quad \forall n \geqslant 1 .
$$

The transformations $f_{\omega}$ will be considered as stochastic perturbations of a deterministic map $f$, in the sense that they will be taken in a suitable neighborhood of $f$ whose size will be determined by the value of $\varepsilon$, see below. We could therefore define a Markov process on $X$ with transition function

$$
L_{\varepsilon}(x, A)=\int_{\Omega_{\varepsilon}} \mathbf{1}_{A}\left(f_{\omega}(x)\right) d \theta_{\varepsilon}(\omega),
$$

where $A \in X$ is a measurable set, $x \in X$ and $\mathbf{1}_{A}$ is the indicator function of the set $A$. A probability measures $\mu_{\varepsilon}$ is called stationary if for any measurable set $A$ we have:

$$
\mu_{\varepsilon}(A)=\int_{X} L_{\varepsilon}(x, A) d \mu_{\varepsilon}(x)
$$

We call it an absolutely continuous stationary measure (acsm), if it has a density with respect to the Lebesgue measure.

Given a map $f: X \rightarrow X$, we will consider two kind of random perturbations. The first one is the additive noise, which corresponds to the family $\left(f_{\omega}\right)_{\omega \in \Omega_{\varepsilon}}$ of random transformations defined by

$$
f_{\omega}(x)=f(x)+\omega \quad \forall x \in X .
$$

In this case each $\omega$ belong to the hypercube $\Omega_{\varepsilon} \subset \mathbb{R}^{d}$ of side $\varepsilon$ centered at zero, and equipped with the measure $\theta_{\varepsilon}=\frac{m}{(\varepsilon)^{d}}$, which is the normalized Lebesgue measure restricted to $\Omega_{\varepsilon}$. For these perturbations, some additional assumptions may be necessary to ensure that the image of each $f_{\omega}$ is included in $X$.

\footnotetext{
${ }^{1}$ In the following when we will refer to a dynamical system $(X, f, \mu)$ we will mean that $f$ is defined on $X$ and preserves the Borel probability measure $\mu$; if we will write $(X, f)$, this will simply correspond to the action of $f$ on $X$.
} 
Notice that for additive noise,

$$
L_{\varepsilon}(x, A)=\theta_{\varepsilon}\left(\omega \in \Omega_{\varepsilon}: f(x)+\omega \in A\right) \leq \theta_{\varepsilon}(A) \leq \frac{m(A)}{\varepsilon^{d}}
$$

which implies that if the stationary measure exists for such random transformations, it is absolutely continuous w.r.t. the Lebesgue measure on the ambient space.

The second kind of random transformations we will consider have been introduced by Lasota and Mackey (see [14], for instance) and correspond to randomly applied stochastic perturbations. They consist in operating an aleatory reset of the state of the dynamical system $(X, f)$ at each failure of a Bernoulli random variable: if $\left(x_{n}\right)_{n \in \mathbb{N}}$ denotes the successive states of such a random dynamical systems, then at each time $n \in \mathbb{N}$ we have $x_{n+1}=f\left(x_{n}\right)$ with probability $(1-\varepsilon)$ and $x_{n+1}=\xi_{n}$ with probability $\varepsilon$, where $\xi_{n}$ is the realization of a random variable with value in $X$. This kind of perturbation corresponds to the family $\left(f_{\omega}\right)_{\omega \in \Omega_{\varepsilon}}$ of random transformations defined by

$$
f_{\omega}(x)=\eta f(x)+(1-\eta) \xi \quad \forall x \in X
$$

where $\omega=(\eta, \xi)$ is a random vector with value in $\Omega_{\varepsilon}=\{0,1\} \times X$. The two components $\eta$ and $\xi$ of $\omega$ are independent and $\eta$ is a Bernoulli variable with the probability of being 0 equal to $\varepsilon$, while $\xi$ is a random variable that we will suppose Lebesgue-uniformly distributed on $X$. The joint distribution $\theta_{\varepsilon}$ of these two components is the product of the Bernoulli measure with weights $(\varepsilon, 1-\varepsilon)$ and the uniform measure on $X$.

In order to obtain the stationary measure $\mu_{\varepsilon}$, let us introduce the random Koopman operator $U_{\varepsilon}$ : $L^{\infty} \rightarrow L^{\infty}$ defined for all $\phi \in L^{\infty 2}$ by

$$
U_{\varepsilon} \phi(x):=\int \phi\left(f_{\omega}(x)\right) d \theta_{\varepsilon} .
$$

Now, if we take two observables $\phi \in L^{\infty}$ and $\psi \in L^{1}$, it easy to check that

$$
\begin{gathered}
\int U_{\varepsilon} \phi(x) \psi(x) d x=\iint \phi\left(f_{\omega}(x)\right) \psi(x) d \theta_{\varepsilon} d x= \\
\varepsilon \iint \phi(x) \psi(y) d x d y+(1-\varepsilon) \int \phi(f(x)) \psi(x) d x=\int \phi(x) P_{\varepsilon} \psi(x) d x,
\end{gathered}
$$

where $P_{\varepsilon}$ is the adjoint operator of $U_{\varepsilon}$, that is the random transfer operator. If we denote $P$ the transfer operator associated to $f$ and $\bar{\psi}=\int \psi(y) d y$, then we have

$$
P_{\varepsilon} \psi(x)=(1-\varepsilon) P \psi(x)+\varepsilon \bar{\psi} .
$$

The stationary measure $\mu_{\varepsilon}$ verifies $\int \phi(x) d \mu_{\varepsilon}=\int U_{\varepsilon} \phi(x) d \mu_{\varepsilon}$ and in our case is given by $\mu_{\varepsilon}=h_{\varepsilon} m$ where $h_{\varepsilon} \in L^{1}$ is a density such that $h_{\varepsilon}=P_{\varepsilon} h_{\varepsilon}$. Such a density exists and is given by [14]:

$$
h_{\varepsilon}=\varepsilon \sum_{k=0}^{\infty}(1-\varepsilon)^{k} P^{k} \mathbf{1} .
$$

\section{Piecewise contracting maps with additive noise}

In this section we study a simple one-dimensional piecewise contracting map perturbed with additive noise. We first recall some known results about the deterministic asymptotic dynamics. Then we give numerical results about their statistical properties when perturbed with additive noise.

\footnotetext{
${ }^{2}$ From now on $L^{1}$ and $L^{\infty}$ will be referred to the Lebesgue measure $m$ and the integral with respect to the latter will be denote as $\int(\cdot) d x$.
} 


\subsection{Deterministic dynamics}

The simplest piecewise contracting map is defined on the unit interval $I=[0,1$ ) (or the circle, also denoted $I)$, by

$$
f(x)=\alpha x+\beta \quad \bmod 1 \quad \forall x \in I,
$$

where $\alpha$ and $\beta$ belong to $(0,1)$. If $\alpha+\beta>1$, then $f$ is discontinuous at the point $\gamma=(1-\beta) / \alpha$ (when considered as a map of the interval) and each restriction of $f$ to one of the intervals $[0, \gamma)$ and $[\gamma, 1)$ is a contraction. The dynamics of this map has been studied in various works, but the most detailed study can be found in [4]. Here we sum up the principal results of interest for our purpose.

To each couple $(\alpha, \beta)$ it is possible to associate a unique rotation number $\rho \in[0,1]$ using a lift of $f$ to a map $F: \mathbb{R} \rightarrow \mathbb{R}$ which stratifies:

$$
\pi \circ F=f \circ \pi
$$

where $\pi(x):=x-\lfloor x\rfloor$ is the projection of $\mathbb{R}$ in the circle $S^{1}$. If we suppose that $F(x+1)=F(x)+1$, then $F$ is uniquely determined modulo an integer additive constant and for any point $x_{0} \in I$ the limit

$$
\rho:=\lim _{n \rightarrow \infty} \frac{F^{n}\left(x_{0}\right)}{n}
$$

exists and does not depend on $x_{0}$. The rotation number $\rho$ is thus a characteristic of the map which depends only on the parameters $\alpha$ and $\beta$. If the rotation number is rational then the attractor $\Lambda:=\bigcap_{n \in \mathbb{N}} \overline{f^{n}(I)}$ of $f$ is composed of a unique periodic orbit of period $q$, where $q$ is the smallest integer such that $q \rho \in \mathbb{N}$. If the rotation number is irrational then $\Lambda$ is Cantor set supporting a minimal dynamics.

Proposition $3.1([4,5])$. The function $\rho$ is continuous in any point $(\alpha, \beta) \in(0,1) \times[0,1)$. For any fixed $\alpha \in(0,1)$ the function $\rho(\alpha, \cdot)$ is non decreasing and its image is $[0,1)$. For any fixed $\beta \in[0,1)$, the function $\rho(\cdot, \beta)$ is non decreasing and its image is $[0, \beta)$. On the other hand, we have that $\rho(\alpha, \beta)=\rho$ if and only if $\beta_{-}(\alpha, \rho) \leqslant \beta \leqslant \beta_{+}(\alpha, \rho)$, where

$$
\beta_{-}(\alpha, \rho)=(1-\alpha)^{2} \sum_{j=0}^{+\infty} \alpha^{j}\lceil(j+1) \rho\rceil \quad \text { and } \quad \beta_{+}(\alpha, \rho)=(1-\alpha)^{2} \sum_{j=0}^{+\infty} \alpha^{j}(\lfloor(j+1) \rho\rfloor+1) .
$$

For the irrational values of $\rho$ we have $\beta_{-}(\alpha, \rho)=\beta_{+}(\alpha, \rho)$ and if $\rho \in \mathbb{Q}$ then $\beta_{-}(\alpha, \rho) \neq \beta_{+}(\alpha, \rho)$. Together with Proposition 3.1, this implies that the graph of $\rho(\alpha, \cdot)$ is a devil staircase, see Figure 1.
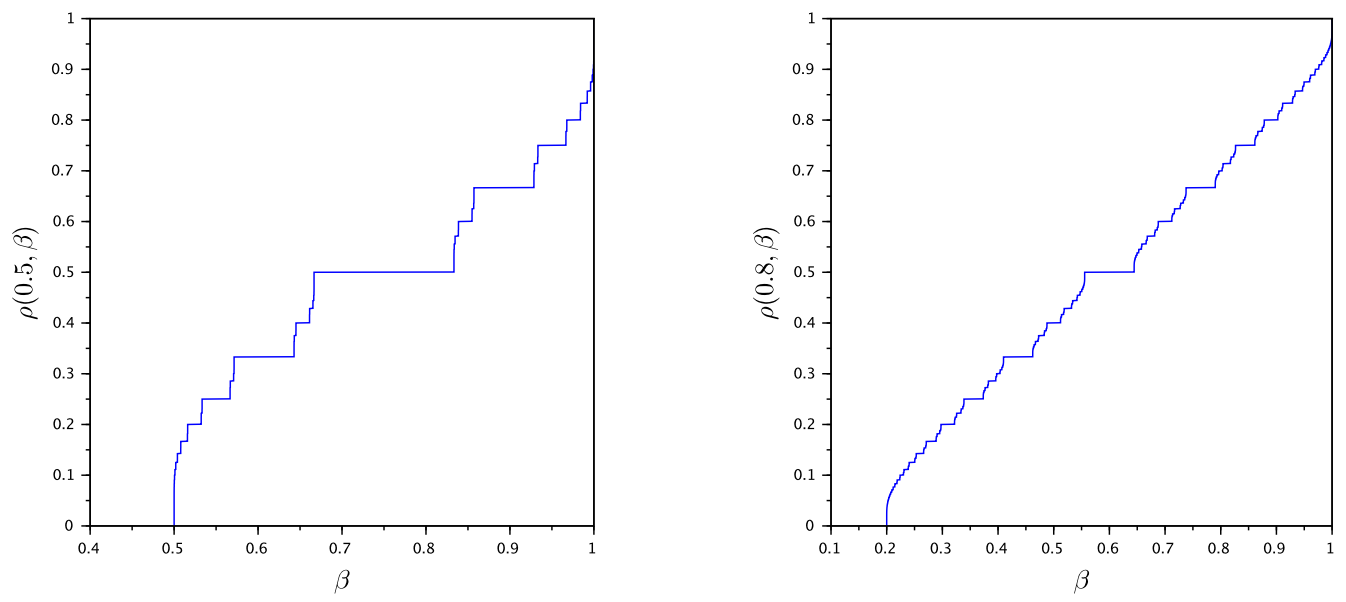

Figure 1: Rotation number as a function of $\beta$, for $\alpha=0.5$ (left) and $\alpha=0.8$ (right).

In the forthcoming subsection we will be interested in studying the system (6) perturbed with additive noise. To achieve this aim we will mostly consider values of $\rho$ for which the difference $\beta_{+}(\alpha, \rho)-\beta_{-}(\alpha, \rho)$ 
is relatively large. By this way, relatively large perturbations of $\beta$ will remain in the interval $\pi_{\rho}:=$ $\left(\beta_{-}(\alpha, \rho), \beta_{+}(\alpha, \rho)\right)$, where $f$ has an attractor composed of a unique periodic orbit (whose period depends on $\rho$ ). In Table 1 we give the center point and the half size of the interval $\pi_{\rho}$ for some selected values of $\rho$ in the case $\alpha=0.8$.

\begin{tabular}{|c|c|c|}
\hline$\rho$ & $\beta_{m}:=\left(\beta_{-}(0.8, \rho)+\beta_{+}(0.8, \rho)\right) / 2$ & $\delta:=\left|\beta_{-}(0.8, \rho)-\beta_{+}(0.8, \rho)\right| / 2$ \\
\hline $1 / 10$ & 0,227 & $3,01.10^{-3}$ \\
$1 / 8$ & 0.245 & $5,04.10^{-3}$ \\
$1 / 4$ & 0.356 & $1,73.10^{-2}$ \\
$2 / 5$ & 0.500 & $1,21.10^{-2}$ \\
$1 / 2$ & 0.599 & $4,44.10^{-2}$ \\
\hline
\end{tabular}

Table 1: Center point $\beta_{m}$ and half size $\delta$ of $\pi_{\rho}$ as a function of $\rho$ for $\alpha=0.8$.

\subsection{Invariant density}

When perturbing with additive noise, we consider random orbits $\left(x_{n}\right)_{n \in \mathbb{N}}$ satisfying

$$
x_{n+1}=f\left(x_{n}\right)+\omega_{n} \quad \bmod 1 \quad \forall n \in \mathbb{N},
$$

where $f(x)=\alpha x+\beta \bmod 1$ for all $x \in I=[0,1)$ and $\alpha, \beta \in(0,1)$. The quantities $\left(\omega_{n}\right)_{n \geq 1}$ are i.i.d. random variables with values in a small interval $\Omega_{\varepsilon}:=[-\varepsilon / 2, \varepsilon / 2]$, where $\varepsilon \in(0,1)$, with a common distribution $\theta_{\varepsilon}$ given by a density $g$, namely $d \theta_{\varepsilon}(\omega)=g(\omega) d \omega$, with $\int_{-\varepsilon}^{\varepsilon} g(\omega) d \omega=1$. We notice that whenever $I$ is the (unit) interval, the strength of the noise, namely $\varepsilon$, should be small enough in such a way the image of $I$ is still in $I$. This problem disappears provided we take the mod-1 operation.

We now add one more assumption on the density $g$, namely we require that the first moment of $g$ be finite. This allows to show that the transfer operator $P_{\varepsilon}$ associated to $(8)$ is weakly constrictive. These kind of operators were introduced by Lasota and Mackay in order to get invariant or stationary measures and as an alternative to the usual theorem of Ionescu-Tulcea Marinescu which in turns uses the spectral gap provided by the Lasota-Yorke inequality. Constrictive operators do not allow to get explicitly statistical properties of the systems, instead they permit to characterize invariant measures for perturbed systems with very low chaotic behaviors.

An operator $P_{\varepsilon}$ is called constrictive if there exists a weakly precompact set $\mathcal{F}$ such that the $L^{1}$ distance between $P_{\varepsilon}^{n} \psi$ and $\mathcal{F}$ goes to zero whenever $n$ goes to infinity and being $\psi$ a $L^{1}$ non-negative function with unit mean, we call $\mathcal{D}$ the space of these functions. These operators enjoy an interesting spectral decomposition, namely: there exists an integer $r$, two subsequences of non-negative functions $g_{i} \in \mathcal{D}$ and $k_{i} \in L^{\infty}, i=1, \ldots, r$, and an operator $Q: L^{1} \rightarrow L^{1}$ such that for all $\psi \in L^{1}, P_{\varepsilon} \psi$ may be written in the form

$$
P_{\varepsilon} \psi(x)=\sum_{i=1}^{r} \lambda_{i} g_{i}(x)+Q \psi(x) \quad \text { where } \quad \lambda_{i}:=\int \psi(x) k_{i}(x) d x .
$$

The functions $g_{i}$ and the operator $Q$ satisfy:

1. $g_{i}(x) g_{j}(x)=0$, for all $i \neq j$, which implies that the densities $g_{i}$ have disjoint supports.

2. For any $i \in \mathbb{N}$, there exists a unique integer $w(i)$ such that $P_{\varepsilon} g_{i}=g_{w(i)}$. Moreover $w(i) \neq w(j)$ when $i \neq j$, where $\{w(1), \ldots, w(r)\}$ is a permutation of $\{1, \ldots, r\}$, so that the operator $P_{\varepsilon}$ permute the densities $g_{i}$.

3. The $L^{1}$ norm of $P_{\varepsilon}^{n} Q \psi$ goes to zero when $n \rightarrow \infty$ and for all $\psi \in L^{1}$.

4. The iterates of the operator have the form

$$
P_{\varepsilon}^{n+1} \psi=\sum_{i=1}^{r} \lambda_{i} g_{w^{n}(i)}+P_{\varepsilon}^{n} Q \psi
$$

where $w:\{1, \ldots, r\} \rightarrow\{1, \ldots, r\}$ is a permutation. 
The last two items justify the appellation of asymptotically periodic given to the sequence $\left(P_{\varepsilon}^{n} \psi\right)_{n \in \mathbb{N}}$. It is interesting to observe that if the noise density $g$ is strictly positive almost everywhere on the circle, then the operator $P_{\varepsilon}$ is also asymptotically stable in the sense that the $L^{1}$ limit of $P_{\varepsilon}^{n} \psi$ is the same independently of $\psi \in \mathcal{D}$. We also observe that the convex combination of the $g_{i}$ given by the spectral decomposition and with equal weights $\frac{1}{r}$ gives a stationary measure.

We also observe that the convex combination of the $g_{i}$ given by the spectral decomposition and with equal weights $\frac{1}{r}$ gives a stationary measure. Now, if $\psi \in \mathcal{D}$ satisfies

$$
P_{\varepsilon} \psi=\sum_{i=1}^{r} \lambda_{i} g_{i} \quad \text { and } \quad P_{\varepsilon}^{r} \psi=\psi
$$

then $\sum_{i}^{r} \lambda_{i}=1$ and $w$ is a ciclic permutation. It follows that

$$
\frac{1}{r} \sum_{j=1}^{r} P_{\varepsilon}^{j}(\psi)=\frac{1}{r} \sum_{i=1}^{r} \lambda_{i} \sum_{j=1}^{r} g_{w^{j}(i)}=\frac{1}{r} \sum_{i=1}^{r} \lambda_{i} \sum_{j=1}^{r} g_{j}=\frac{1}{r} \sum_{j=1}^{r} g_{j}
$$

is an invariant density. Therefore, if we obtain a numerical estimation of a density $\psi$ satisfying (10), we do not need to know the densities $g_{i}$ of the spectral decomposition to obtain an estimation of the invariant density.

We will suppose that the i.i.d random variables $\left(\omega_{n}\right)_{n \geq 1}$ of $(8)$ are uniformly distributed in $[-\varepsilon / 2, \varepsilon / 2]$, with $\varepsilon \in(0,1)$, and thus the common distribution is given by the density $g=\mathbf{1}_{[-\varepsilon / 2, \varepsilon / 2]}$, where $\mathbf{1}_{A}$ refers to the indicatrice function of the set $A$. We study numerically the iterations of the transfert operator associated to the random dynamical system (8), in particular the asymptotic periodicity of the sequence $\left(P_{\varepsilon}^{n} \psi\right)_{n \in \mathbb{N}}$, where $\psi=\mathbf{1}$ is the uniform density on the circle.

In Figure 2, 3 and 4, we plot for $n>300$ some numerical estimations of $P_{\varepsilon}^{n} \mathbf{1}$. The parameters of $f$ are fixed and equal to $\alpha=0.8$ and $\beta=0.5$, which corresponds to a value of $\beta$ in the middle of the plateau $\rho=2 / 5$ (see left panel of Figure 1 and Table 1). The attractor of $f$ is thus a periodic orbit of period 5 which position depends continuously on $\beta$.
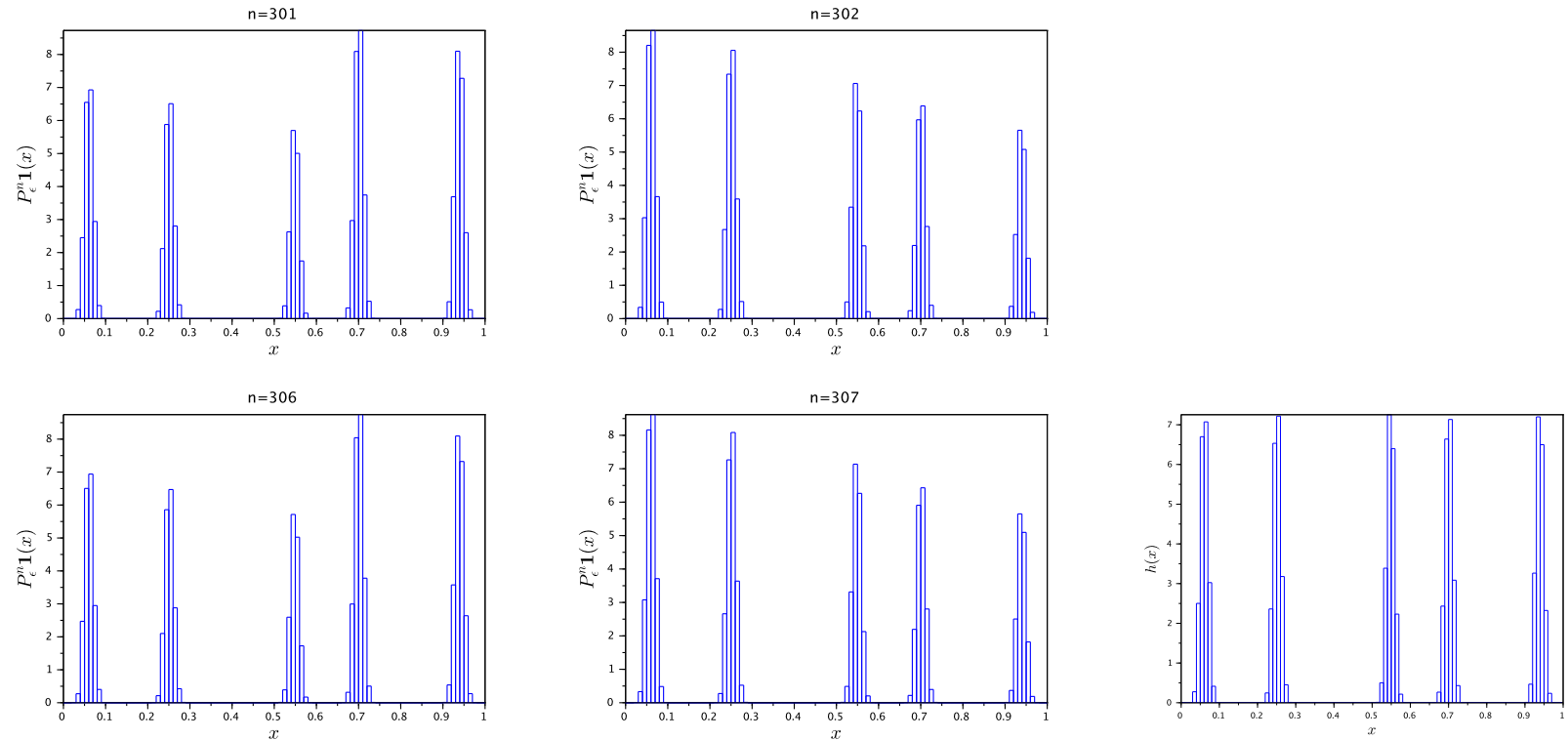

Figure 2: Histogram of $P_{\varepsilon}^{n} \mathbf{1}$ for $n=301,302,306$ and 307, with $\alpha=0.8, \beta=0.5$ and $\varepsilon=0,02$. Right panel: the corresponding invariant density, denoted $h$.

We consider first a small noise of amplitude $\varepsilon=0.02$. As expected, we observe in Figure 2 that the support of the densities is contained in a neighborhood of the periodic orbit of $f$. The figure also 
shows that $P_{\varepsilon}^{301} \mathbf{1}$ and $P_{\varepsilon}^{302} \mathbf{1}$ are very similar to $P_{\varepsilon}^{306} \mathbf{1}$ and $P_{\varepsilon}^{307} \mathbf{1}$, respectively. This shows that $\left(P_{\varepsilon}^{n} \mathbf{1}\right)_{n \in \mathbb{N}}$ is asymptotically periodic of period 5. We conclude that in presence of small noise the period of the deterministic attractor of $f$ was conserved trough the period of an attracting density of the random transfer operator. Now, we can obtain the invariant density by using formula (11) (Right panel if Figure 2).
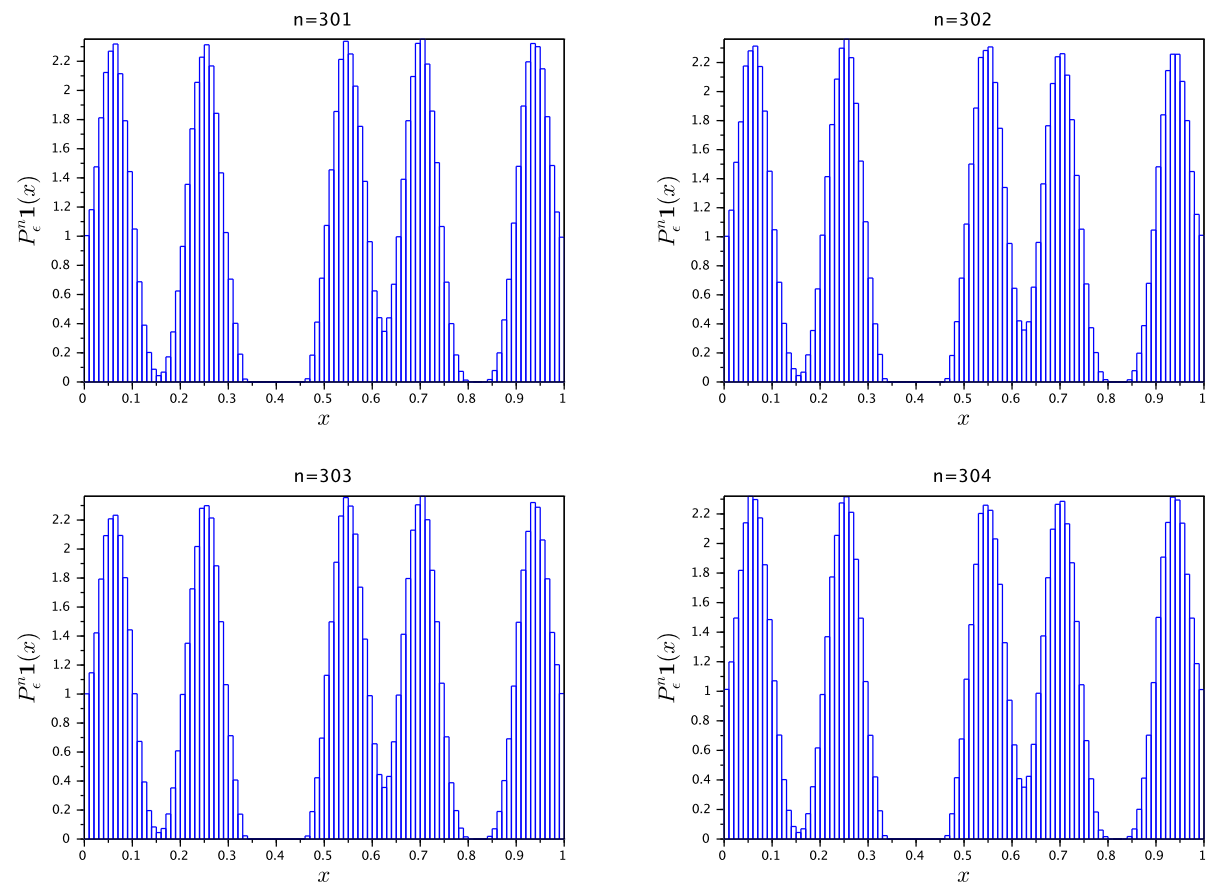

Figure 3: Histogram of $P_{\varepsilon}^{n} \mathbf{1}$ for $n=301,302,303$ and 304, with $\alpha=0.8, \beta=0.5$ and $\varepsilon=0,07$.

In Figure 3, we increase the noise to $\varepsilon=0.07$. Now, the support of the densities has 2 connected components: one in the interval $[0.46,0.8]$ and another one in the interval $[0.84,1] \cup[0,0.34]$ (which in the circle is a connected set). It is tempting to conjecture that these two components are each one the support of two densities $g_{1}$ and $g_{2}$ of formula (9), and thus to deduce that $\left(P_{\varepsilon}^{n} \mathbf{1}\right)_{n \in \mathbb{N}}$ is asymptotically periodic of period 2. However, there is no significative difference between the four consecutive iterations of $P_{\varepsilon}$ shown in Figure 3 and we can conclude that $\left(P_{\varepsilon}^{n} \mathbf{1}\right)_{n \in \mathbb{N}}$ converges to a fixed density, which is estimated by those of Figure 3.
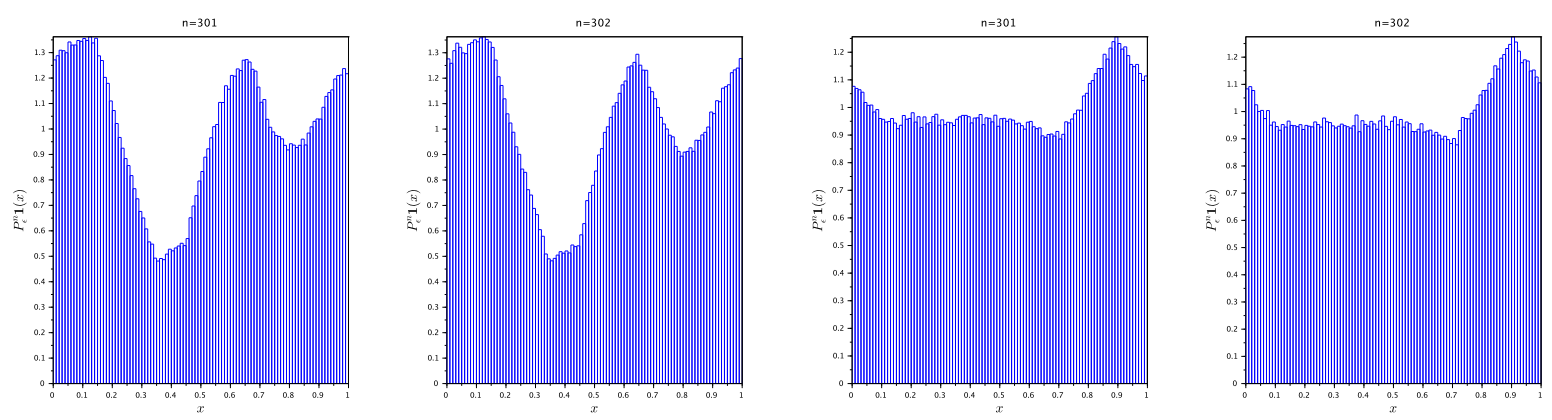

Figure 4: Histogram of $P_{\varepsilon}^{n} \mathbf{1}$ for $n=301$ and 302, with $\alpha=0.8, \beta=0.5$. Left panel: $\varepsilon=0.3$, right panel $\varepsilon=0.8$.

In Figure 4, we consider the strong noises $\varepsilon=0.3$ and $\varepsilon=0.8$. In both cases, the support of the 
densities is the full circle and $\left(P_{\varepsilon}^{n} \mathbf{1}\right)_{n \in \mathbb{N}}$ is asymptotically periodic of period 1 .

The system (8) can also be written as:

$$
x_{n+1}=\alpha x_{n}+\beta_{n} \quad \bmod 1 \quad \forall n \in \mathbb{N},
$$

where $\left(\beta_{n}\right)_{n \geq 1}$ is a sequence of i.i.d random variables defined by $\beta_{n}=\beta+\omega_{n}$ and uniformly distributed in the interval $I_{\beta, \varepsilon}:=[\beta-\varepsilon / 2, \beta+\varepsilon / 2]$.

Let $\rho \in[0,1]$ and denote $\pi_{\rho}:=\left(\beta_{-}(\rho), \beta_{+}(\rho)\right)$. We recall that $f$ has a rotation number equal to $\rho$ iff $\beta \in \pi_{\rho}$. Since $\beta_{n}$ is uniformly distributed in $I_{\beta, \varepsilon}$, we have that

$$
\mathbb{P}\left(\beta_{n} \in \pi_{\rho}\right)=\frac{l\left(\pi_{\rho} \cap I_{\beta, \varepsilon}\right)}{l\left(I_{\beta, \varepsilon}\right)}=\frac{l\left(\pi_{\rho} \cap I_{\beta, \varepsilon}\right)}{\varepsilon},
$$

where $l$ denotes the Lebesgue measure. In Figure 2, 3 and 4 we consider $\alpha=0.8$ and $\beta=0.5$. For these values of parameters $\beta$ belongs to the plateau $\pi_{2 / 5}$ which extremities are given by $\beta_{-}(0.8,2 / 5)=\beta-\delta$ and $\beta_{+}(0.8,2 / 5)=\beta+\delta$, where $\delta=1,21 \cdot 10^{-2}$ (see left panel of Figure 1 and Table 1 ). It follows that in Figure 2, where $\varepsilon=0.02<2 \delta$, we have $I_{\beta, \varepsilon} \subset \pi_{\rho}$ and $\mathbb{P}\left(\beta_{n} \in \pi_{2 / 5}\right)=1$. The system (8) is therefore the composition of maps which each attractor is a period 5 orbit. This orbit is different for each map, but its points depend continuously on $\beta$. It is therefore expected to observe a period 5 attractor for the transfert operator. On the other hand, in Figure 3 we have $\varepsilon=0.07>2 \delta$, which implies now $\pi_{\rho} \subset I_{\beta, \varepsilon}$ and $\mathbb{P}\left(\beta_{n} \in \pi_{2 / 5}\right)=2 \delta / \varepsilon \simeq 0.35$. It follows that almost $2 / 3$ of the realizations of $\beta_{n}$ are outside the plateau $\rho=2 / 5$, which result in the loss of the asymptotic periodicity (different from period 1 ) of the transfert operator observed in Figure 3. Finally, in Figure 4 we have $\varepsilon=0.3$ and $\varepsilon=0.8$ and $\mathbb{P}\left(\beta_{n} \in \pi_{2 / 5}\right) \simeq 0.08$ and $\mathbb{P}\left(\beta_{n} \in \pi_{2 / 5}\right) \simeq 0.03$, respectively. In these case, more that $90 \%$ of the realizations of $\beta_{n}$ do not belongs to $\pi_{2 / 5}$. The random orbits of (8) are therefore obtained by composition of maps which in a great majority have a periodic attractor which is not of period 5. Actually, the union of these attractors covers a large part of the phase space.

Now, we may ask if the noise can change the period of the attracting density of the transfert operator. More precisely, for $\alpha$ and $\beta$ fixed, if we suppose that $\left(P_{\varepsilon}^{n} \mathbf{1}\right)_{n \in \mathbb{N}}$ is asymptotically periodic of period $q$, we ask if it exists $\varepsilon^{\prime}$ such that $\left(P_{\varepsilon^{\prime}}^{n} \mathbf{1}\right)_{n \in \mathbb{N}}$ is asymptotically periodic of period $q^{\prime} \notin\{1, q\}$. As suggested by our analysis of Figures 2, 3 and 4, we will suppose that the asymptotic periodicity of $P_{\varepsilon}$ is related to the proportion of realizations of the random variables $\beta_{n}$ in a given set $\pi_{\rho}$.

Let $p \in[0,1], \varepsilon \in[0,1]$ and $\rho \in[0,1]$. Then, one can show that

$$
\mathbb{P}\left(\beta_{n} \in \pi_{\rho}\right) \geqslant p \quad \text { iff } \quad l\left(\pi_{\rho}\right) \geqslant \varepsilon p \quad \text { and } \quad \beta \in\left[\beta_{-}(\alpha, \rho)+\varepsilon\left(p-\frac{1}{2}\right), \beta_{+}(\alpha, \rho)-\varepsilon\left(p-\frac{1}{2}\right)\right] .
$$

We deduce that for any $\varepsilon$, if $p \geqslant 0.5$ then $\beta \in \pi_{\rho}$. In other words, if we want to impose that more than $50 \%$ of the realizations of $\beta_{n}$ belong to $\pi_{\rho}$, and to force by this way a given period (uniquely determined by $\rho$ ) for the attracting density of $P_{\varepsilon}$, we must chose the parameter $\beta$ of the deterministic map $f$ in $\pi_{\rho}$. Therefore, if $\beta \in \pi_{\rho}$, it is not possible to obtain at least $50 \%$ of the realizations of $\beta_{n}$ in $\pi_{\rho^{\prime}}$, with $\rho^{\prime} \neq \rho$, by mean of a suitable choice of $\varepsilon$.

After the above analysis it appears difficult to change to a period different from 1 the period of the attracting density of the transfert operator by addition of uniform noise. However, the relation (13) is specific to symmetric uniform noise. If now we consider that the random variables $\beta_{n}$ are uniformly distributed in the interval $J_{\beta, \varepsilon}:=[\beta, \beta+\varepsilon]$ (i.e $\omega_{n} \in[0, \varepsilon]$ ) then (13) becomes

$$
\mathbb{P}\left(\beta_{n} \in \pi_{\rho}\right) \geqslant p \quad \text { iff } \quad l\left(\pi_{\rho}\right) \geqslant \varepsilon p \quad \text { and } \quad \beta \in\left[\beta_{-}(\alpha, \rho)-\varepsilon(1-p), \beta_{+}(\alpha, \rho)-\varepsilon p\right],
$$

It follows that, for $p$ and $\rho$ fixed, it is possible to chose $\beta \leqslant \beta_{-}(\alpha, \rho)$, thus not in $\pi_{\rho}$, and to find an $\varepsilon$ such that $\mathbb{P}\left(\beta_{n} \in \pi_{\rho}\right) \geqslant p$, provided the following relation between the size of $\pi_{\rho}$ and its distance to $\beta$ holds:

$$
l\left(\pi_{\rho}\right) \geqslant \frac{p}{1-p}\left(\beta_{-}(\rho, \alpha)-\beta\right) .
$$

Actually, if (15) is satisfied then

$$
\mathbb{P}\left(\beta_{n} \in \pi_{\rho}\right) \geqslant p \quad \forall \varepsilon \in\left[\frac{1}{1-p}\left(\beta_{-}(\rho, \alpha)-\beta\right), \frac{l\left(\pi_{\rho}\right)}{p}\right] .
$$



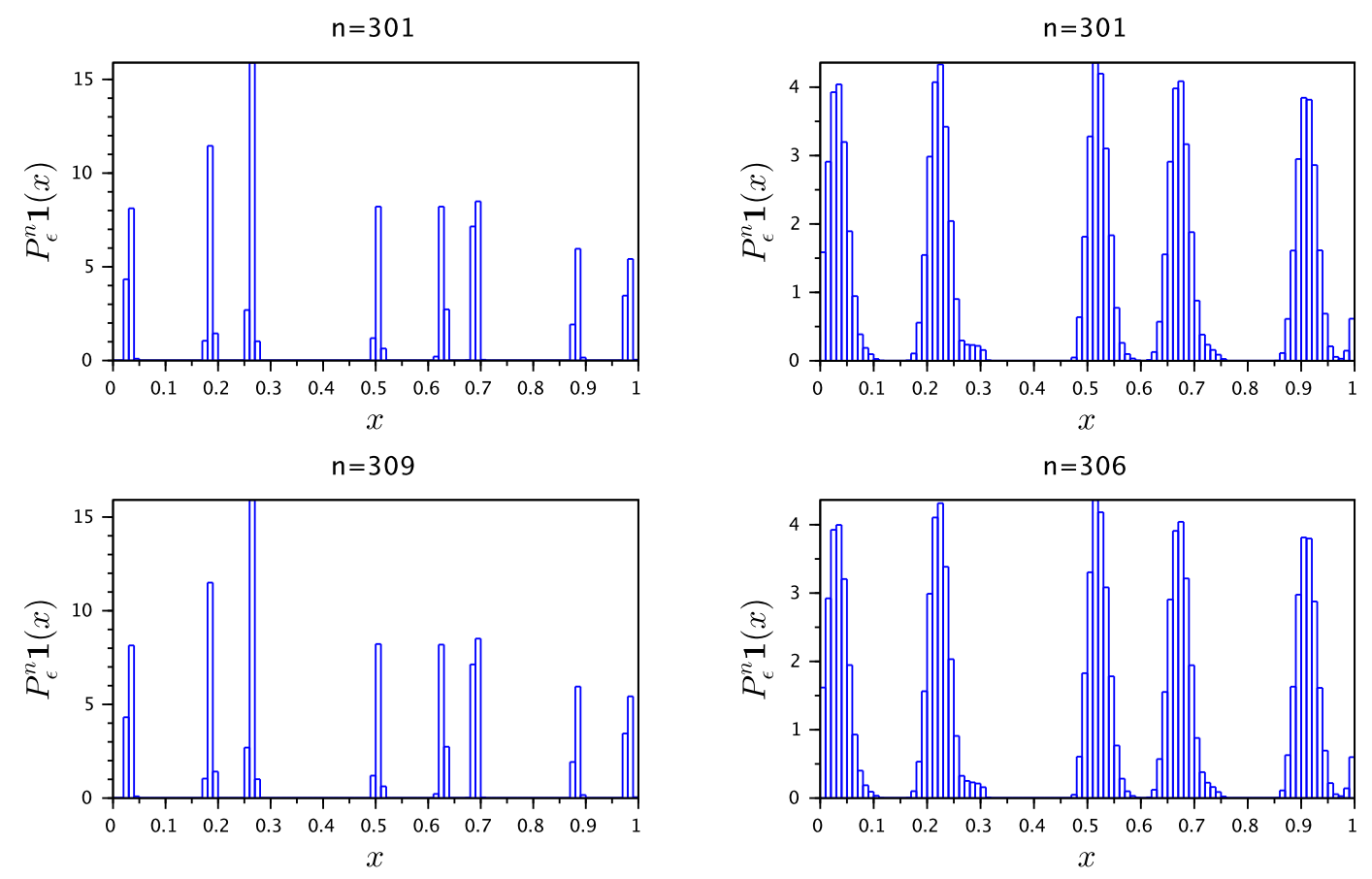

Figure 5: Histogram of $P_{\varepsilon}^{n} \mathbf{1}$ for $\alpha=0.8, \beta \simeq 0.475$. Left: $\varepsilon \simeq 0.0076$, upper panel $n=301$, lower panel $n=309$. Right: $\varepsilon \simeq 0.0366$, upper panel $n=301$, lower panel $n=306$.

Since (15) can be satisfied with $p>1 / 2$, we can modify the period of the attracting density of the transfert operator by addition of noise, as done in Figure 5. To obtain Figure 5 , we chose $\beta \simeq 0,475 \in \pi_{\frac{3}{8}}$, which corresponds to a period 8 attractor for the deterministic map and for the transfert operator perturbed with small noise (left pannel). For this value of $\beta$, the condition (15) holds for $\rho=2 / 5$ if $p=0,65$ (or less). Now taking $\varepsilon=l\left(\pi_{\frac{2}{5}}\right) / p \simeq 3,66.10^{-2}$, we have $\mathbb{P}\left(\beta_{n} \in \pi_{\frac{2}{5}}\right) \geqslant 0,65$ and we observe a period 5 attractor for $P_{\varepsilon}$ (right panel).

\subsection{Extreme value statistic}

The study of extreme value statistics is typically concerned with the limiting distributional behaviour of partial maxima of a stochastic process, which is determined by the likelihood of the occurrence of abnormally high observations (or exceedances of high thresholds) along the time series. In [11, 12], motivated by [3], the study of extreme value statistics is formally linked to the recurrence properties of deterministic systems, namely Hitting/Return Time Statistics, for specially designed observable functions defined on the phase space.

For well behaved systems, i.e., systems that loose memory sufficiently fast, typically, the waiting time between exceedances (or returns to target sets) is exponentially distributed, which is consistent with the observation of an asymptotic classical extreme value distribution for the partial maxima. As shown in [13], in the presence of periodic behaviour, as observed at periodic points for deterministic systems, the pattern of the exceedances is affected by clustering, whose intensity is measured by the extremal index (EI). In fact, the EI can be seen as the inverse of the average cluster size, being that no clustering means an EI equal to 1 and heavy clustering an EI close to 0 . We refer to [10] for further reading and references.

Only very recently the study of extreme values and hitting/return times was carried for randomly perturbed dynamical systems. We mention in particular the very recent papers $[1,16,15,17]$. The first one uses an annealed approach, i.e., the laws are obtained by integrating over all possible values of the noise, while the latter use a quenched approach, where one is concerned with the extremal behaviour for a.e. realisation of the noise. 

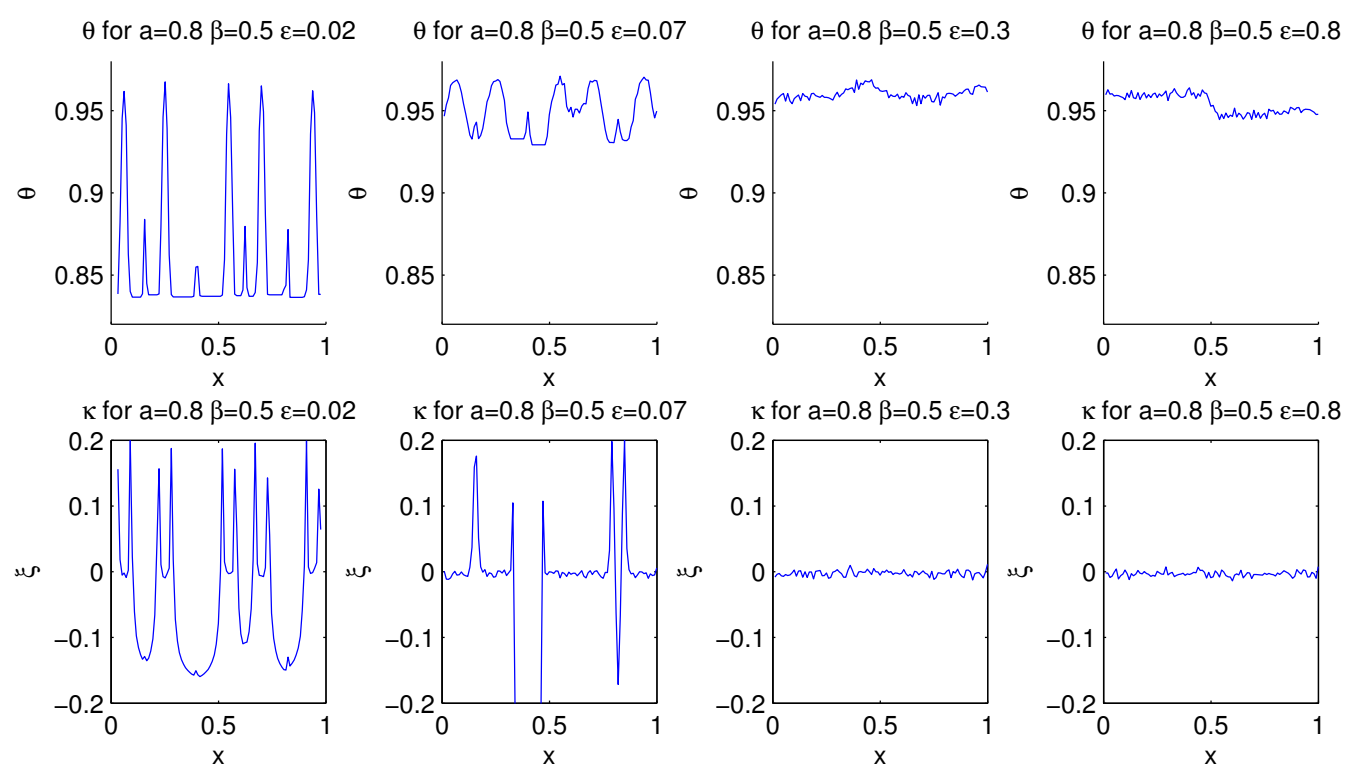

Figure 6: Extreme value analysis for $P_{\epsilon}^{n} 1$. Upper panels: extremal index $\theta$. Lower panels: shape parameter $\kappa$. From left to right noise is increasing as specified in the titles of each subplot.

Until now, the existence of an EVL has been established, essentially, for expansive maps and rotations of the circle perturbed with additive noise $[1,8]$. We have shown [7] that piecewise contracting maps can also admit an EVL under RASP. Here we discuss the case of additive noise.

Contrarily to the case of RASP, we are not able to produce rigorous results about the rate of decay, but a distribution on the whole circle of random orbits of arbitrarily chosen initial conditions suggest the presence of only one fixed point for $P_{\varepsilon}$ and therefore mixing. This seems the case in Figure 4 and, to a lesser extent, in Figure 3. Indeed, the GEV statistics effectuated for the usual observable shows a pretty good convergence towards the Gumbel's law for strong noise. In Figure 6, we show the dependence of the EI $\theta$ and the shape parameter $\kappa$ on the choice of the points $x$ of the phase space around which we study the returns, when the noise is taken on a symmetric interval.

The upper panels report the results for $\theta$ and the lower panels for $\kappa$. From left to right the noise increases and take the values $0.02,0.07,0.3$ and 0.8 as in the experiments of the previous subsection. The comparison with the histograms in Figures 2, 3 and 4 shows that when $x$ is visited often enough the convergence to the Gumbel law is good $\kappa \simeq 0$ and the extremal index approaches 1 . When the point is not visited enough, the extremal index is lower than 1 and the shape parameter largely deviates from 0 . Increasing the noise corresponds to have a more uniform visit of the phase space and is reflected by a better convergence to the Gumbel law and by a more uniform extremal index $\theta$ (see right panels of Fig. 6)

\section{Baker map}

let us consider the Baker's map $f$ defined on the unit square $[0,1] \times[0,1]$ iteratively for $n \geq 1$, by

$$
\begin{aligned}
& x_{n+1}=\left\{\begin{aligned}
\gamma_{a} x_{n}, & \text { for } y_{n}<\alpha \\
\frac{1}{2}+\gamma_{b} x_{n}, & \text { for } y_{n}>\alpha
\end{aligned}\right. \\
& y_{n+1}=\left\{\begin{aligned}
\frac{1}{\alpha} y_{n}, & \text { for } y_{n}<\alpha \\
\frac{1}{1-\alpha}\left(y_{n}-\alpha\right)+\gamma_{b} x_{n}, & \text { for } y_{n}>\alpha
\end{aligned}\right.
\end{aligned}
$$

We analyse the effect of the RASP and the additive noise on this map. To avoid to exit from the square we apply the mod 1 operator after having applied the additive noise perturbation. The parameters used 
for the experiment are the following: $\gamma_{a}=1 / 5, \gamma_{b}=1 / 4, \alpha=1 / 3$. We want to analyse the existence of an extremal index $\theta<1$ on the boundary of the rectangles describing the invariant density.

\subsection{Invariant density}

Let's look at the attractors we obtain for the additive and the RASP perturbation of the Backer map. In Figure 7 the left panel refers to the additive noise, the right panels to the RASP perturbation and the noise intensity is increasing from upper to lower panels. By looking at the two different perturbations, strong differences are evident. For the additive noise the bands of the baker attractor are recognizable and they get fatter for increasing noise intensity although, even for strong noise intensities, we have unexplored region of the phase space. For the RASP perturbation the invariant measure consists of the usual Baker attractor, superimposed with a uniform noise whose level depends on the noise intensity $\epsilon$. If we transpose this example to photography, additive noise would be add to a picture if the camera or the objective are moving randomly while taking the picture, additive noise would be more the results of underexposition, the lower the light, the higher the noise.

These results are translate in terms of invariant density in the upper panel of Figure 8. For the additive noise we recover the density peaks corresponding to the location of the bands. For the RASP perturbation the relative weight of the real baker dynamics and the uniform level added via RASP is visible for different noise intensities is a set of point uniformly distributed everywhere on the rectangle. In other words, the additive noise makes the rectangles bigger until the invariant density is almost uniform (yellow curves corresponding to $\epsilon=0.05$ ). For the RASP perturbation what changes is the relative weight of the uniform density with respect to the rectangles.

\subsection{Extreme value statistics}

Since the definition of boundaries depends on the noise intensity, for the EVL analysis we take several values $z$ for the observable $\phi$ in the rectangle [0 1], and consider distances on the $x$ variable only. All the analysis follows exactly the procedure described in [9]. The shape parameter $\kappa$ of the GEV distribution is shown in the central panels of Figure 8. We know that in the complete stochastic case, for the choice of the $\log$ weighting function for $\phi$, a GEV fit should return $\kappa=0$. By comparing the values of $\kappa$ with the densities shown in the upper panels, we observe that around the points such that the density is large enough, $\xi \simeq=0$. This is even clearer for the lowest noise intensity (blue) for which convergence $\kappa \rightarrow 0$ is bad everywhere except in the band of the baker attractor. On the contrary for the largest noise intensity (yellow) we approach the stochastic limit and $\kappa=0$ almost everywhere. Convergence is very poor for the RASP perturbation and low level noise at the boundaries between the attractor and the region of uniform noise.

Finally we present the computation of the extremal index $\theta$ for all the cases considered (lower panels of Figure 8). Let us focus on the azure line $(\epsilon=0.02)$ and look at the additive noise (left panel) only. We compare $\theta$ with the density reported in the upper panel. The boundaries of the attractor for this level of noise are localized around $x=0.8$ where the azure curve decrease and then interrupts (no points) for $x>0.82$. If we look this area, we see that: for the points in the Baker attractor, $\theta<1$ and we have values around $\theta=0.95$, when we move outside the attractor, towards the boundaries $\theta \simeq 0.82$ the extremal index decreases coherently with the theoretical results presented. If we look at the violet curve and compare it with the density in the upper panel, we find a similar behaviour with the extremal index $\theta$ decreasing when the density increases although for this noise level the attractor is already continuous. The value of $\theta=0.95$, i.e. not one as predicted by the theory, is a finite size effect. As we have verified the values of $\theta$ tends to 1 for increasing bin length (not shown).

Now let us focus on the RASP perturbation whose results for $\theta$ are reported in the lower right panel. As for $\kappa$ also the extremal index is very different with respect to the one computed for the additive noise. The only region where $\theta<1$ appears is at the point $x=2 / 3$. Here the extremal index decreases to the value $\theta=1 / 3$ for all the noise intensities, although it recovers faster to one for higher noise levels. This is not a finite size effects as we have verified by increasing the bin length. This behavior is coherent with the theoretical results which implies that for observables with a maximum at a periodic point then the EI 

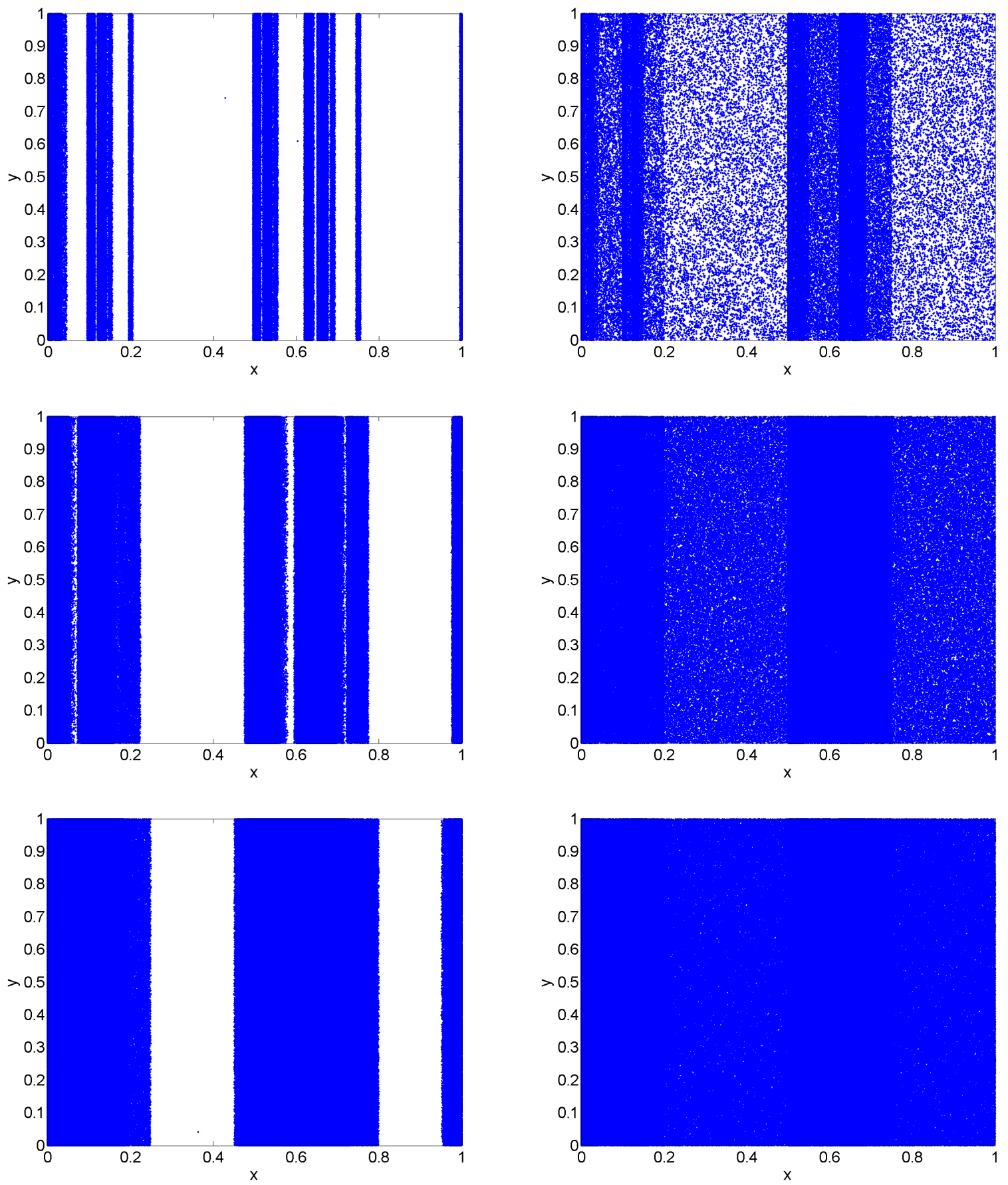

Figure 7: Baker attractors for additive noise (left) and RASP perturbation (right). Upper panels: $\epsilon=0.01$, Central panels: $\epsilon=0.05$, Lower panels: $\epsilon=0.1$ 
should be less than 1 and a combination of the EI of the original map and the $\eta$. However, one should note that there are no theoretical results asserting that the EI is less than 1 at such periodic points for the original map, the only result in that direction is in a recent paper by Carvalho et al [2] but it only applies to toral automorphisms.

Acknowledgement. All authors acknowledge the Isaac Newton Institute for Mathematical Sciences, where this work was initiated during the program Mathematics for the Fluid Earth. DF is supported by the ERC Grant A2C2 (No. 338965). JMF was partially supported by FCT grant SFRH/BPD/66040/2009 (financed by the program POPH/FSE) and also by CMUP, which is financed by FCT (Portugal) through the programs POCTI and POSI, with national and European structural funds, under the project PEstC/MAT/UI0144/2013. JMF and SV are also supported by FCT (Portugal) project PTDC/MAT/120346/2010, which is financed by national and European structural funds through the programs FEDER and COMPETE. PG was supported by CONICYT project Anillo ACT1112. SV was supported by the ANR- Project Perturbations, by the project Atracción de Capital Humano Avanzado del Extranjero MEC 80130047, CONICYT, at the CIMFAV, University of Valparaiso, by the projet MODE TER COM supported by Region PACA, France and by the project BREUDS, Brazilian-European partnership in Dynamical Systems.

\section{References}

[1] H. Aytac, J. M. Freitas, and S. Vaienti. Laws of rare events for deterministic and random dynamical systems. Trans. Amer. Math., 367 (11) 8229-8278 (2015).

[2] M. Carvalho, A. C. M. Freitas, J. M. Freitas, M. Holland, and M. Nicol, Extremal dichotomy for uniformly hyperbolic systems, Dynamical Systems, 2015.

[3] P. Collet, Statistics of closest return for some non-uniformly hyperbolic systems, Ergodic Theory Dynam. Systems, 21 (2) 401-420 (2001)

[4] R. Coutinho, Dinâmica simbólica linear. PhD Thesis, Technical University of Lisbon, 1999.

[5] Coutinho R, Fernandez B, Lima R and Meyroneinc A 2006 Discrete time piecewise affine models of genetic regulatory networks Journal of Mathematical Biology 52 (4) 524-570

[6] D. Faranda, J.M. Freitas, P. Guiraud, S. Vaienti, Sampling local properties of attractors via extreme value theory, Chaos, Solitons and Fractals, 74, 55-66, (2015).

[7] D. Faranda, J.M. Freitas, P. Guiraud, S. Vaienti, Extreme Value Theory for Piecewise Contracting Maps with Randomly Applied Stochastic Perturbations, Stochastics and Dynamics, in press.

[8] D. Faranda, J. Freitas, V. Lucarini, G. Turchetti, S. Vaienti, Extreme Value Statistics for Dynamical Systems with Noise, Nonlinearity, 26, 2597-2622, (2013)

[9] D. Faranda, V. Lucarini, G. Turchetti, S. Vaienti, Numerical convergence of the block-maxima approach to the Generalized Extreme Value distribution, J. Stat. Phys, 145 (5), 1156-1180, (2011)

[10] J. M. Freitas, Extremal behaviour of chaotic dynamics, Dyn. Syst. 28 (3) 302-332 (2013)

[11] A. C. M. Freitas, J. M. Freitas, and M. Todd, Hitting time statistics and extreme value theory, Probab. Theory Related Fields 147 (3-4) 675-710 (2010)

[12] A. C. M. Freitas, J. M. Freitas, and M. Todd, Extreme value laws in dynamical systems for nonsmooth observations, Journal of Statistical Physics 142 (1) 108-126 (2011)

[13] A. C. M. Freitas, J. M. Freitas, and M. Todd, The extremal index, hitting time statistics and periodicity, Adv. Math., 231 (2012), 2626-2665.

[14] Lasota, A., and Mackey, M. C. (1994). Chaos, fractals, and noise: stochastic aspects of dynamics (Vol. 97). Springer. 

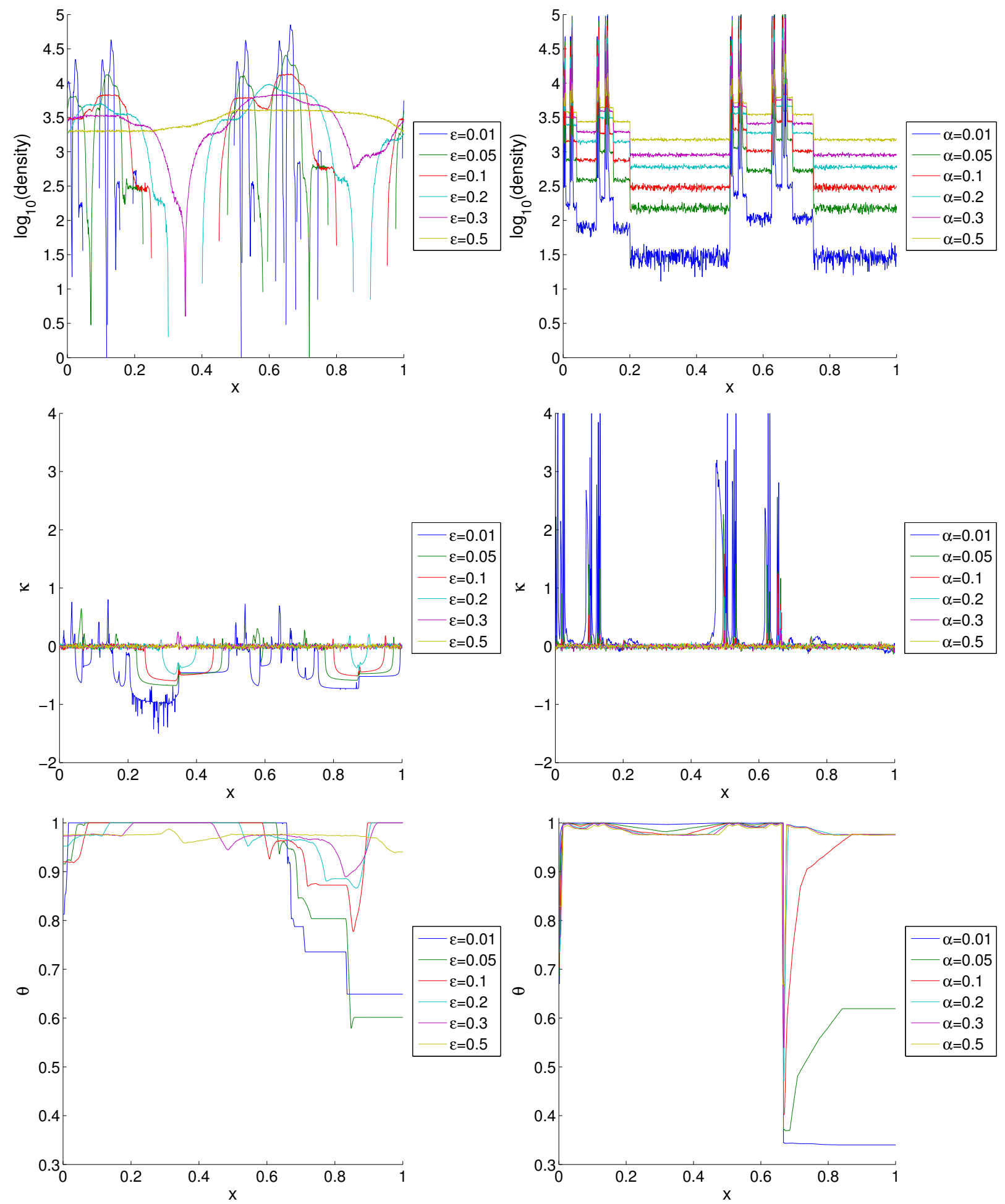

Figure 8: Upper panels: invariant density for the $x$ variable on the Baker map. Central panels: shape parameter $\kappa$ for the GEV distribution. Lower panels: extremal index $\theta$. Left: additive noise. Right: RASP. 
[15] J. Rousseau, Hitting time statistics for observations of dynamical systems, Nonlinearity 27 (9) $2377-$ $2392(2014)$.

[16] J. Rousseau, B. Saussol and P. Varandas, Exponential law for random subshifts of finite type, Stochastic Process. Appl. 124 (10) 3260-3276 (2014).

[17] J. Rousseau and M. Todd, Hitting times; Random dynamical systems; Exponential law; Extremal index, J. Stat. Phys. 161 (1) 131-150 (2015). 